\title{
Aprendizagem dos adolescentes sobre cuidado de si mesmos: o retorno aos ensinamentos parentais
}

\author{
Adolescents' learning about caring for themselves: \\ the return to parental teachings
}

\section{Adriana María Ramírez Barrientos}

Universidad de Antioquia. Medellín, Antioquia, Colômbia. ORCID: 0000-0002-7330-6671. adriana.ramirez@udea.edu.co

RESUMO | INTRODUÇÃO: Pesquisa realizada com adolescentes em que o cuidado de si mesmo é identificado como uma construção nas relações parentais. Objetivo: Analisar o aprendizagem de cuidado de si mesmo num grupo de adolescentes e a maneira em que hospedam as orientações parentais em suas vidas cotidianas. METODOLOGIA: qualitativo-etnográfico; as técnicas de coleta de dados foram por meio de grupos focais e entrevistas semi-estruturadas. Os participantes eram adolescentes com idades entre 16 e 19 anos. RESULTADOS: para os adolescentes o ensinado pelos pais está enquadrado nas normas e pautas para evitar os enganos na tomada de decisões; considerar e aceitar estes ensinos, é caminhar para o próprio conhecimento de si mesmos. DISCUSSÃO: As relações parentais estão condicionadas pelo poder e controle que os pais exercem sobre seus filhos através da regulação da norma, sendo um aspecto de fixação e tensão para os adolescentes. Viver a experiência de ser adolescente, de reconhecer o erro, é o que os define como sujeitos e recreia possibilidades para tomar decisões e atuar em frente às circunstâncias quotidianas. CONSIDERAÇõES FINAIS: Os adolescentes aprendem através de suas próprias experiências a cuidar de si mesmos. Os ensinos recebidos pelos pais formam seu sistema de valores, o qual é resignificado por meio da conduta que promovem na cada experiência vivida; esta resignificación contribui novas formas de pensar e atuar, definindo sua identidade e a maneira como assumem sua vida.

PALAVRAS-CHAVE: Adolescente. Família. Parentalidad. Relações familiares.
ABSTRACT | INTRODUCTION: Research conducted with adolescents where identifies the care of himself same like construction in the parental relations. OBJECTIVE: analyze the learning of self care of adolescents in late stage and the way in which narratives of parents are taken into account in the daily life. METHOD: qualitative- ethnographic; focus groups and semi-structured interviews were carried out. The participants were adolescents between the ages of 16 and 19. RESULTS: For adolescents taught by parents is framed in the rules and guidelines to avoid mistakes in decision making; To consider and accept these teachings is to walk to the self-knowledge of themselves. DISCUSSION: Parental relationships are conditioned by the power and control that parents exert on their children through the regulation of the norm, being an aspect of restraint and tension for adolescents. To Live the experience of being a teenager, of recognizing the error, is what defines them as subjects and recreates possibilities to make decisions and act in the face of everyday circumstances. CONCLUSION: Teenagers learn through their own experiences to take care of themselves. The teachings received by the parents form their system of values, which is resignified through the conduct that they promote in each lived experience; This resignification brings new ways of thinking and acting, defining its identity and how it assumes its life.

KEYWORDS: Adolescent. Family. Parenting; family relationships. 


\section{Introducción}

La adolescencia como etapa del ciclo vital humano se caracteriza por la búsqueda y conformación de una identidad, en la mayoría de los casos, parte de un modelo externo. Dicho modelo es elegido por los adolescentes en concordancia con las necesidades psicológicas, afectivas y sociales que estén experimentando. La respuesta que estos jóvenes puedan asumir frente a cada circunstancia, viene de unos aprendizajes pre-existentes de la infancia y que son transmitidas desde el seno familiar. Díaz y Mejía, (2018) describen que son necesarias ciertas aptitudes para integrar el desarrollo humano del individuo, se agrupan en tres categorías: a. habilidades sociales: comunicación asertiva y empatía en las relaciones interpersonales; b. habilidades cognitivas: aquellas que denotan la toma de decisiones, el pensamiento crítico y creativo; y c. las habilidades de control de emociones: prácticas que permiten al adolescente conocer sus sentimientos y reconocer los momentos adecuados en los que debe tener un manejo apropiado de los mismos.

Autores como Berger (2016), confieren que el desarrollo de la adolescencia comienza con la pubertad, comprendida como la etapa en que se presenta un rápido crecimiento físico y maduración sexual, que pone fin a la infancia para dar lugar a una persona con la capacidad física y fisiológica de llevar a cabo la reproducción humana. En esa medida, la respuesta hormonal no solo tiene efecto sobre el cuerpo sino sobre las emociones; las manifestaciones de ira o tristeza se combinan con las reacciones y esta cadena, activa comportamientos y conductas que en algunos casos, resultan ser riesgosos para la salud y la integridad del adolescente.

Si bien es cierto que los jóvenes se transforman en hombres y mujeres iniciando con su desarrollo físico y sexual, sobreviene después de este primer cambio la maduración cerebral. Las características sexuales que diferencian la masculinidad o la feminidad, permiten que tanto hombres como mujeres, experimenten un aumento del interés sexual a medida que madura el cuerpo y aumentan los niveles hormonales, por tanto, las conductas sexuales y los pensamientos de estos adolescentes, estarán fuertemente influídos por la cultura y el deseo de experimentar. En lo referente al desarrollo cerebral, Güemes-Hidalgo, González-Fierro \& Vicario, (2017) describen que "se ha observado que las zonas del cerebro adolescente que buscan la recompensa, se desarrollan antes que las zonas relacionadas con la planificación y el control emocional" (p. 11); lo cual significa que la experimentación, exploración y conductas de riesgo en esta etapa del desarrollo, son más de carácter normativo que patológico. También afirman que el cerebro de los adolescentes tiene gran capacidad para adaptarse a nuevos cambios, lo que explicaría las posibilidades reales de modificar y adoptar nuevas conductas.

En efecto la adolescencia trae consigo el soporte de un ideal en el que el joven espera encontrar la razón de su lugar en el mundo para consolidar su proyecto de vida. Si las bases recibidas para construir su identidad son deficientes, el establecimiento como sujeto será difuso y carente de estructura, lo cual traería como resultado, pobres mecanismos de respuesta ante las demandas y necesidades que deberá enfrentar en cada acontecer diario (Berger, 2016).

Este proceso resulta ser determinante en el ciclo familiar, dado que es la familia el dispositivo social donde se brinda protección y se transmiten culturalmente aquellos hábitos y directrices para el buen desarrollo y conservación de las relaciones intersubjetivas (Rojas, Méndez, y Álvarez, 2016). En esta misma línea, Alzate (2014) describe la familia como la expresión primera y fundamental de la naturaleza social del hombre.

Las bases enseñadas por los padres en el hogar, consideran valores, normas, pautas sociales, prinicipios, derechos y deberes como la forma más apropiada de llevar a cabo la crianza; el resultado esperado es que sean hijos aceptados y productivos para la sociedad. Uno de los elementos preponderantes en el rol de ser padre, es precisamente el cuidado de los hijos. Es de considerar que históricamente el cuidado ha sido definido como una práctica que el hombre ha construido como producto de sus experiencias y significados, sin embargo, las familias son diversas y por lo tanto crecen hijos diversos, los cuales son atravesados por diferentes contextos que inciden en sus conductas, decisiones y relaciones (Rojas, Méndez y Álvarez, 2016).

Cuidar es un asunto natural que se encuentra relacionado con los comportamientos, actitudes y sistema de valores de cada sujeto en su cotidianidad; por ello, cuidar de los hijos parte de los aprendizajes 
de las propias experiencias y del modelo parental asumido por los padres; Blanco y Licona (2016) lo definen como una estructura mediada por la cultura que determina los patrones de convivencia familiar y las responsabilidades de cada uno de sus miembros. Considera además, las diversas manifestaciones que surgen en la interacción según las características propias de los sujetos que la conforman, con el fin último de conservar la cohesión y el bienestar general.

En esa medida la familia se convierte para el adolescente en el primer agente social en el que viven experiencias de cuidado físico y emocional. Son los padres los que derivan en los hijos conductas y hábitos para el cuidado propio, por lo tanto estos aprendices suman elementos que con el pasar de las vivencias, moldean y definen como atributos de comportamiento y toma de decisiones. En lo concerniente a la práctica e interacción familiar, una vez se conforma un sistema, -en este caso la familiase debe conocer la función de cada estructura y la manera cómo estas se relacionan entre sí y a la vez mirar cómo influye el ambiente social en ese sistema, es decir, cómo se ve inmerso el sistema (familia) en el supra sistema (ambiente). El resultado de esa interrelación interna y externa del sistema familiar, determina su propia dinámica en relación a los patrones, aprendizajes y conductas de sus miembros (Castillo, Sibaja, Carpintero y Romero-Acosta, 2015).

El sistema familiar desde la perspectiva evolutiva, ha sido producto de grandes transformaciones estructurales dada la pluralidad en su conformación, los roles y las funciones que de manera diversa han asumido ambos géneros según las necesidades y demandas internas del sistema. Es por ello que más allá de la tipología que la defina, las transiciones previas, la composición y sus dinámicas, constituyen el capital cultural que se hereda a los hijos y posteriormente la forma en que éstos lo replican en sus interacciones con otros sujetos (Villalba, 2015; Díaz-Alzate y Mejía-Zapata, 2018).

Algunos de los estudios internacionales y nacionales hallados, han analizado el comportamiento de los adolescentes en términos de conductas de riesgo, las cuales parecieran ser interpretadas como una ruptura en el cuidado de sí mismo. Estas comprenden: inicio de relaciones sexuales a temprana edad, maternidad adolescente, consumo de sustancias psicoactivas, suicidio, violencia juvenil, entre otras. Situaciones que alteran no solo la vida cotidiana, proyecto de vida y salud del adolescente, sino la relación parental en términos afectivos y comunicativos, generando consecuencias nefastas para el vínculo filial (Cogollo \& Gómez-Bustamante, 2013; Campo, Silva, Meneses, Castillo, Navarrete, 2004; Plasín y Goicochea, 2012)

En esta misma línea, la mayoría de los estudios se orientan hacia el conocimiento, identificación y comprensión de causas y efectos del comportamiento adolescente en dirección a la alteración y el padecimiento físico (Contini, Coronel, Lacunza, Mejail \& Caballero, 2014). Otros autores como Hernández, (2018) describen asuntos de igual trascendencia y mencionan situaciones más allá del contexto privado familiar, que influyen en el cuidado de los jóvenes y que tienen relación directa con otros agentes socializadores como: los mecanismos de exclusión social y género, las diferencias entre la regulación y cumplimiento de la legislación que los protege y los reafirma como ciudadanos de derecho, el desbordante consumo social, los modelos de producción y las tendencias en el mercado mundial, la educación y las oportunidades laborales, aspectos que han transformado el concepto de cuidado de las nuevas generaciones y por lo tanto, los mecanismos de respuesta ante sus necesidades individuales y familiares.

En lo que respecta al concepto de cuidado de sí, abarca al ser humano más allá de una condición de riesgo para salud. El cuidado de sí surge como una propuesta por el respeto hacia el propio ser y la autocomprensión, expresada en la actitud que el sujeto debe adoptar frente a la vida y el mundo (Hallal, 1998). Desde la perspectiva Foucaultiana (Foucault, 2001), se afirma que es la forma en que cada individuo se asume en el mundo, se relaciona con el otro y con el entorno a través de acciones y conductas que le otorgan nuevos aprendizajes y significados. Toma en cuenta el proceder y la manera en que cada sujeto recrea estrategias basadas en experiencias y aprendizajes, que modifican su comportamiento y a su vez, definen su identidad.

El propósito de este estudio se basó en las siguientes preguntas: ¿Cómo es la relación entre adolescentes y padres y la forma en que esta influye en su comportamiento y toma de decisiones? ¿Qué consideran los adolescentes que han aprendido de los padres para su propia vida? y ¿Cómo definen los adolescentes las diversas formas que han aprendido para cuidarse? 
En segundo lugar, se centró en la construcción del conocimiento comprensivo de cómo los adolescentes interpretan y significan el cuidado de sí mismos ante las experiencias acontecidas, la lectura que hacen de los aprendizajes obtenidos de los padres y la forma en que esas herramientas son significativas en sus vivencias. Y por último, esta investigación pretendió construir un referente bibliográfico orientador de nuevas propuestas investigativas en el área social, para nutrir otras miradas relacionadas con adolescentes y el cuidado de sí mismos.

\section{Metodología}

Este estudio cualitativo con enfoque etnográfico, está orientado hacia la comprensión de las experiencias que viven los adolescentes y los aprendizajes para el cuidado de símismos, según la lectura que hacen de las enseñanzas parentales. De esta manera espacialidad, corporeidad, temporalidad y relacionalidad como existenciales básicos, permiten al investigador, conocer de manera detallada y rigurosa lo que hacen los adolescentes -prácticas de cuidado- y lo que significan dichas prácticas en su vida cotidiana -perspectivas y aprendizajes- (Restrepo, 2016).

El trabajo investigativo estaba orientado hacia la población adolescente, por lo que se optó por una Institución Educativa, dada la posibilidad concreta de obtener una mayor participación por parte de la población de interés. Una vez se tuvo la aprobación para el trabajo de campo por el Comité de Ética de la Facultad de Medicina de la Universidad de Antioquia(Medellín septiembre de 2015), se contactó al Rector de un Colegio público de básica secundaria (Bachillerato) ubicado en el municipio de Medellín (Antioquia), quien dio su autorización para que se llevara a cabo el proceso investigativo.

Como criterios de selección fueron considerados: adolescentes con edades entre los 16-19 años, matriculados en el colegio, que estuviesen cursando último grado de formación ( $11^{\circ}$ grado). Adicionalmente que vivieran con alguno de sus padres y que voluntariamente desearan participar del estudio. Para lograr la participación se llevó a cabo un encuentro con dos grupos de estudiantes a quienes se les explicó detalladamente el asentimiento libre como participantes, sumado permiso de sus padres para los que fuesen menores de 18 años. Una vez se conoció el número de menores de edad que aceptaron participar, se hizo contacto telefónico con el padre o madre para explicar la razón y finalidad de la investigación en la que participarían sus hijos, y a través de ellos, se les hizo llegar el formato de consentimiento para su firma respectiva. Se aclararon dudas al respecto y se explicó tanto a los padres como a los adolescentes participantes que la información obtenida sería con fines exclusivamente académicos, rigurosamente resguardada, para mantener la privacidad y su anonimato, adicionalmente que dicha información, no sería utilizada con fines evaluativos o informes comportamentales para los padres o el colegio.

Esta investigación tomó en cuenta los lineamientos éticos según la Regulación ética en investigación con seres humanos en Colombia, la cual está regida por la Resolución 008430 del Ministerio de Salud- 1993. Se acogen todas las directrices internacionales para realizar investigación con seres humanos.

En lo relacionado a las técnicas de investigación utilizadas para acceder a la voz de los participantes, fueron realizados:

Grupos focales. Se efectuaron tres encuentros con los grupos focales, conformados por un número entre 4 y 5 participantes. Durante el primer encuentro, los participantes realizaron unas escarapelas en los que anotaron un seudónimo, lo que permitió organizar la transcripción de los datos e identificar posteriormente los testimonios expresados por cada participante. En cada uno de los encuentros se efectuó una didáctica diferente (talleres, conversatorios y encuestas), por lo tanto, para cada momento, con el apoyo del audio y el video se obtuvo información valiosa de las experiencias narradas por los participantes con relación al tema.

Una vez terminado los grupos focales, toda la información fue transcrita en su totalidad, se utilizaron códigos en letras y números para identificar a cada participante y de esta forma se construyó el primer protocolo obtenido de esta herramienta de recolección. Es de anotar que desde ese mismo momento se dio inicio al análisis de los datos, con la lectura detallada del protocolo transcrito, se identificaron unidades significativas que dieron paso a la formación de códigos que posteriormente, se fueron alimentados con otros datos similares; 
una vez culminada esta primera fase, se procedió a realización de las entrevistas.

Entrevistas semiestructuradas: Para la realización de esta segunda técnica de recolección, se obtuvo la participación de 3 adolescentes ( 2 hombres y 1 mujer) a razón de que los tiempos en que se llevaron a cabo, coincidió con el cierre del año académico y fueron éstos los que aceptaron ser entrevistados. Cada participante fue entrevistado en una biblioteca externa al colegio en fechas diferentes, de tal manera que fuera resguardada su identidad; la grabación de las entrevistas fue transcrita por la misma investigadora, información con la que se construyó un segundo protocolo y una vez analizado, se continuó con la construcción de los códigos identificados anteriormente; se hizo necesario, un segundo momento de entrevistas para lograr la saturación de los datos.

Con la construcción de los protocolos, esta información fue llevada al Software Atlas Ti-v7 para su análisis, el cual permitió categorizar los patrones de los datos obtenidos y de esta forma, buscar los diferentes significados temáticos. Este trabajo arrojó códigos, que dieron paso a la conformación final de tres dimensiones significativas: (i) el cuidado en la relación parental, (ii) cuidado de sí mismo y (iii) las normas de los padres.

Para una mayor comprensión de los resultados, se nombran los relatos de los adolescentes identificados con la letra " $A$ " que significa la letra inicial de la palabra adolescente, seguido de una letra del abecedario en minúscula para determinar el orden del participante y por último la edad en años; ejemplo: A.b-18 años.

\section{Resultados y Discusión}

La familia es un sistema social conformado por seres humanos para atender necesidades como las afectivas, biológicas y psicológicas; estructura una red de relaciones entre los miembros y determina sus dinámicas de funcionamiento. Según el ciclo vital en el que se encuentre la familia, la red relacional podrá verse modificada tantas veces sea necesario, esto es lo que probablemente ocurre en las familias con hijos adolescentes. Aspectos como la socialización, las relaciones de pareja, el consumo de alcohol y sustancias psicoactivas, así como la sexualidad, se desarrollan en la medida de lo que el adolescente haya obtenido de su familia, ya sea que tienda a imitar lo aprendido o que opte por ir en contravía de los hábitos familiares.

Comprender las relaciones familiares entre padres y adolescentes, parte de la premisa planteada por Bertalanffy (1992), quien considera que todo organismo viviente es un sistema, conformado por partes y procesos que interactúan entre sí, en un contexto determinado. Comprender la lectura sistémica de la familia, implica entender la relación entre el comportamiento de sus individuos y sus respuestas, focalizado en las interacciones, roles, formas de funcionamiento y organización.

En este orden de ideas, el sistema familiar del adolescente, está en una búsqueda permanente de acuerdos y restablecimiento de normas, dado que es en este momento del ciclo vital familiar en el que suele haber mayor presencia de conflictos familiares, situaciones de violencia entre padres e hijos y conductas de riesgo, lo que aumentan la posibilidad de mayor disociación familiar (Muñoz y Graña, 2001; Güemes, et al., 2017).

\section{El cuidado en la relación parental}

Las situaciones que diariamente viven los adolescentes, toman diversas interpretaciones desde lo social, comunicacional, conductual y relacional. Los adolescentes consideran que es una etapa de dificultades y desacuerdos con los padres y en otras ocasiones, de apoyo y cuidado. Al respecto una de las adolescentes refiere que:

[...] el papá y la mamá, son una referencia para uno, son los encargados de cuidarnos; ellos son el eje central

[...] (A.a-17 años)

Para los adolescentes, el cuidado recibido de los padres es motivado por la suma de cambios y nuevas situaciones a las que se ven enfrentados y en las que pueden presentarse dificultades en la toma de decisiones. Esta es una razón por la cual algunos padres, asumen su rol de cuidadores brindando consejos y buscando espacios para el diálogo.

\section{[...] Mi papá una vez me dijo una frase muy bonita: -mi abuelo fue alguien, mi papá fue alguien mejor que mi abuelo, yo intenté ser alguien mejor que mi papá y tú tienes que ser alguien mejor que yo [...] (A.b-18 años).}


A través de la comunicación los sujetos se vinculan en un conocimiento social y cultural, como elemento fundante de identidad en el entorno donde interactúan (Medina, Álvarez y Hernández, 2017). Por tal razón, la forma en que las personas hablan, escriben, leen y escuchan, hace parte de todo un sistema convencional que las conecta socialmente y crea bajo esos estándares, realidades y relaciones entre unos y otros. En palabras de Watzlawick (1985) se afirma que "toda conducta en una situación de interacción, tiene un valor de mensaje, es decir, es comunicación, se deduce que por mucho que uno lo intente, no puede dejar de comunicar" (p.50).

Para Medina, et al., (2017) la comunicación familiar refleja los patrones de interacción entre sus miembros, la manera en que intercambian mensajes informativos o normativos y a su vez, la cohesión y conexión entre los sujetos. Es preponderante que exista en el grupo familiar una comprensión mutua, centrada en el correcto desempeño de los roles y la realización de las tareas propias de cada uno de los miembros; esto quiere decir que la comunicación emitida por los padres debe ser clara, directa y suficiente y que los receptores estén en disposición y apertura para evitar distorsiones. La comunicación es elemento indispensable para identificar y resolver los problemas familiares.

En los momentos de interacción, algunos de los jóvenes consideran que sus padres son representativos y reciben su apoyo incondicional. $Y$ así no siempre ambas figuras estén presentes, (padre y madre) el que lo está, asume la responsabilidad de acompañar, por ende, de brindar cuidado.

[...] para mí mi mamá es muy importante, porque mi papá murió cuando yo tenía 4 años, entonces siempre he estado con mi mamá, sólo mi mamá ha estado presente [...] (A.c-18 años).

De esta forma, el vínculo afectivo es preponderante en el mantenimiento de las relaciones parentales como una medida de protección y seguridad y aunque podría pensarse social y culturalmente que es más necesario en la etapa de la infancia, a medida que los hijos crecen y se hacen autónomos, creen que siguen necesitando el afecto y el apoyo de los padres.

[...] no por el hecho de estar en esta edad ya grande, quiere decir que no necesite de mis papás, por el contrario, ellos siguen siendo muy importantes para mí y sé que hacen lo que hacen porque me quieren y me cuidan [...] (A.a-17 años).

[...] es que cuando uno cuida de alguien o algo, no es tanto por deber, sino porque uno siente que le nace querer cuidar del otro porque le interesa lo que le pase

[...] (A.d-18 años).

Al respecto, Henao (2012) considera que cada comportamiento de un miembro de la familia, es potencializado por los demás, de tal forma que el cuidado por el otro, representa una manera de interés genuino e inocuo como representación del afecto y el amor filial.

[...] me llevo muy bien con mi papá, ha sido una relación de confianza, porque él sabe quién soy yo; confía en lo que hago y decido para mi vida, así a veces sea una equivocación, él me aconseja y siempre quiere lo mejor para mí [...] (A.a-17 años).

Sin embargo en ciertas situaciones, las relaciones con los padres suelen ser de mucha tensión; esto lo ven representado cuando éstos no creen en sus palabras y acciones y las fundamentan en ideas o hechos que los hacen dudar de su capacidad de decisión.

[...] hay momentos en que la relación con mi mamá es muy tensa, pasa un problema y yo me ofendo porque ella se pone en contra mía o siente algo que la hace desconfiar de mí [...] (A.d-18 años).

En ese sentido, la interacción entre padres e hijos está condicionada por la secuencia comunicacional que existe entre ellos. Por tanto, las experiencias que los padres consideran como errores de sus hijos, dificultan la comprensión de la "causa-efecto" del mensaje comunicado en esa conducta, dado que la causa siempre será diferente para el padre y para el hijo, al igual que el efecto; lo cual contrasta con las habilidades que ambos tengan para transmitir mensajes claros y congruentes en términos resolutivos y efectivos. Disponer de habilidades positivas en la comunicación, facilita la expresión espontánea de sentimientos positivos y negativos que puedan surgir como resultado de la interacción (Medina, et al.; 2017; Gracia \& Musitu, 2000).

Podría deducirse que las conductas y rutinas que viven los adolescentes al interior de sus familias, orientan sus prácticas cotidianas y tienen como punto de partida la relación con el otro. Esa relación podría definirse como una técnica que exige ejercitarse, 
aprender "el arte de vivir"- el cual parte de la lectura diaria de las acciones y su conexión con su entorno. Estas prácticas que realizan los adolescentes buscan establecer una forma de ser, de pensar y comportarse según su criterio y esto lo definen como su verdad. En palabras de Foucault (1990), esto hace referencia a las tecnologías, comprendidas como las matrices de la razón práctica, en la cual emerge la tecnología del yo, comprendida como la base que:

permite a los individuos efectuar por cuenta propia o con la ayuda de otros, cierto número de operaciones sobre el cuerpo y su alma, pensamientos, conducta, o cualquier forma de ser, obteniendo así una transformación de sí mismos con el fin de alcanzar cierto estado de felicidad (p. 48).

Esta diversidad de prácticas, está dada por una serie de técnicas que los hombres utilizan para entenderse a sí mismos y de esta manera, determinar sus momentos de verdad, razones y lógicas de sus acciones.

En esa medida, las relaciones y las normas familiares construyen una serie de representaciones y formas de resolver los problemas que cotidianamente surgen de la interacción. En el momento en que ocurren las crisis y entran en tensión los supuestos y reglas entre los padres y los hijos, es cuando las posturas adolescentes y parentales deben considerar las capacidades del sistema para reajustar el punto desequilibrante. Implica, considerar las libertades de opinión y pensamiento de ambos lados, de permitir la expresión significante de cada experiencia vivida como un mecanismo de confianza y validación del otro. Apelar por la concertación en lugar de la imposición, es para los adolescentes dejar de ser un simple observador y cumplidor de órdenes, es ser reconocido como participante activo de la palabra, en un sentido manifiesto del cuidado y acompañamiento de los padres en este proceso vital de sus vidas. En la misma línea, la validación que los adolescentes hagan de la experiencia de sus padres como hechos de verdad y de enseñanza, será una respuesta de reconocimiento al afecto y a la relación parental.

Dado lo anterior los adolescentes construyen sus formas de ser, estar, verse y sentirse en relación con otros. Se vuelven lectores del otro, de los padres, por lo tanto en la interacción ambos se recorren como autores y lectores en permanente elaboración.
Si bien los adolescentes reconocen la familia como el primer espacio social de aprendizaje del cuidado de sí mismos, consideran que en la etapa en la que ellos se encuentran, los padres buscan mantener de cierta forma el control de las conductas. Esta cultura que viene desde la infancia, pareciera adquirir un segundo momento para los jóvenes en esta etapa: suman cuestionamientos, opiniones y oposiciones a ciertos lineamientos parentales, por lo tanto, dejan de ser aprendizajes "forzados" para edificar nuevas ideas y formas del cuidado de sí mismos.

El cuidado de sí implica identificar qué es lo trasgresor y qué es la libertad; qué es lo que reafirma la vida, y qué es lo que reprueba la sociedad. Por tanto, es posible afirmar que la experiencia del error puede generar reflexiones y aprendizajes que los adolescentes pueden resignificar en torno al cuidado de sí, pues solo en ese punto, sería posible afirmar la vida como un proceso de subjetivación de la experiencia vivida. Es ahí donde puede palparse aquello que realmente lo potencia o lo degrada como sujeto (Foucault, 1978; 1983).

La vivencia es la única manera en que los adolescentes se acercan al aprendizaje de su propio cuidado. La experiencia del descuido, surge como la posibilidad de generar una mayor consciencia de su cuerpo, emociones, significados y aprendizajes del propio cuidado de sí mismo (Foucault, 1978; 1983; 1990; 1994).

\section{Cuidado de sí mismo}

Como se ha venido explicando, cuidar de sí mismo es una labor permanente que exige al sujeto conectar todos los recursos -físicos, éticos y emocionales- para mantener en equilibrio su pensar, sentir y actuar. Sin embargo, para los adolescentes, este asunto es algo que va más allá del control y el equilibrio que puedan tener de su cuerpo y pensamiento en ciertas circunstancias. La necesidad de experimentar en cierta medida el error, la equivocación, es la manera como algunos logran un aprendizaje consciente y significativo y un conocimiento de sí mismos.

\section{[...] para uno cuidarse, uno se tiene que conocer, si uno no se conoce ¿entonces qué va a cuidar?[...]}

(A.b-18 años). 
Nombrar el término "sí mismo" en relación con el autoconocimiento, implica conocer en primer lugar las fortalezas, elementos de apoyo para hacer las cosas bien. El conocimiento en su expresión básica, podría considerarse como una necesidad elemental que rodea la existencia del ser humano. A través del conocimiento es posible descubrir la razón y el significado de "algo". La manifestación de lo oculto en el mundo en que se vive. Conducido al plano del sujeto, el cuidado de sí mismo es sin duda el conocimiento de un cierto número de reglas, conductas o principios que son verdades para quien las asume y prescriben su forma de ser y actuar como individuo (Foucault, 1990).

Los adolescentes consideran que cuidar de sí mismos, está definido como tener bienestar, es decir, el estado que se logra cuando se piensa claramente lo que se va a hacer y en esa medida, llevarlo a cabo. Ese "saber pensar", que los orienta a reflexionar sobre las cosas que ellos creen son convenientes para sus vidas.

\section{[...] para mí las fortalezas consisten en saber cómo me voy a cuidar y en lo que tengo que invertir mi tiempo y mis capacidades [...] (A.e-18 años).}

Por lo tanto, podría pensarse que las fortalezas surgen de un proceso de aprendizaje que vive el adolescente como experiencia de su propio cuidado. Cuando es capaz de evidenciar el error y generar un pensamiento distinto en torno a él, se vuelve estratégico en su actuar, dispone de sus propias capacidades y valores para asumir el control de sí mismo.

Por tal razón, las decisiones que los adolescentes toman en torno al cuidado de sí mismos consideran dos aspectos fundamentales, a mencionar: las circunstancias o experiencias vividas y la puesta en escena de aquellas bases aprendidas en el hogar y en otros espacios de socialización. Lo que acontece en últimas, es que ellos son los que conducen sus decisiones y acciones para alcanzar o no su propio bienestar.

Foucault (2001), citando la filosofía platónica menciona que el "sí mismo" es el conocimiento que está más allá de las capacidades, del alma y las pasiones; es en cierto modo una cuestión metodológica, que conduce al sujeto a comprender que es él mismo quien se ocupa de sí como objeto; es a la vez sujeto y objeto. En esta misma línea, esta dualidad va dirigida a conocerse a sí mismo, a descubrir las deficiencias del ser en una forma reflexiva. Es una tarea que en consideración, dirige al sujeto a ocuparse del alma (psykhe) a fin de que llegue a ser lo mejor posible para el autoconocimiento en un sentido pleno de su ser.

Esa relación consigo, implica disponer de todo los recursos propios y articularlas para el cuidado de sí mismo, -una de las grandes reglas de conducta constituida en la época del mundo grecorromanopor tanto para Foucault (2001), estas técnicas se encuentran en todas las culturas, bajo diferentes formas y manifestaciones; descrito en palabras de un adolescente:

[...] el cuidado va muy ligado al respeto, pienso que uno se cuida cuando se conoce muy bien y cuida de algo o alguien, cuando siente un respeto muy grande, cuando entiende la diferencia entre lo bueno y lo malo [...]

(A.d-18 años).

En el aprendizaje dado por las experiencias, los jóvenes también resaltan la importancia de las debilidades, no solo como algo negativo, sino como una oportunidad de corregirse y disponerse para un cambio de conducta y de pensamiento. Una debilidad podría comprenderse como el momento en que se pierde el bienestar.

\section{[...] Yo creo que una debilidad es cuando aun sabiendo} que algo no es conveniente para uno, uno lo termina haciendo y ahi es cuando uno se equivoca y mete las patas [...] (A.f-16 años)

Fernández (2018), menciona que en la actual modernidad, mencionar el cuidado de sí mismo o retornar al principio del sí, se ha volcado hacia la instalación de ciertos mecanismos de resistencia que en parte definen la subjetivación del individuo; esta subjetividad está ligada a la construcción permanente de la identidad, la adopción de creencias que determinan las acciones propias de cada persona y en esa medida, la interacción con otros como principio de equivalencia subjetiva. Visto así, cada ser humano está invitado a enfrentar sus propios problemas, a examinarse en su pensamiento y acción cotidiana. Es por ello que los adolescentes consideran que las decisiones son importantes en sus vidas y cuando son las correctas, es decir, cuando se piensan para elegir, pueden prever las consecuencias y asumirlas con la responsabilidad que se debe, independiente de la razón u opinión que puedan tener sus padres al respecto; en esa medida, sería una práctica positiva de cuidado de sí mismo. 
[...] el cuidado es como una sensación de ser responsable, de saber lo que uno hace o deja de hacer para sentir que está haciendo las cosas bien [...]

(A.g-17 años).

Todo este proceso de cuidado viene acompañado de los aprendizajes obtenidos de las experiencias vividas en el colegio, el hogar, grupo de amigos y de sus propias equivocaciones. Refieren que así como han sido cuidados por otros, ellos también brindan cuidados a las personas que son importantes para ellos, por lo tanto el cuidado trasciende sus vidas y las de otros. En esa medida, lo transmitido por los padres y otras personas significativas, de cierta forma constituye su sistema de valores y modo de pensar; son elementos que permanecen arraigados y sobresalen ante el proceso reflexivo de esas situaciones consideradas como equivocaciones.

Ese proceso que los lleva a tener control sobre sí mismo o posesión de sí mismo, es referido por Foucault (1990), como el "gobierno de sí", el poder que el sujeto tiene sobre sí mismo para agenciar sus pensamientos y prácticas para cuidarse a sí mismo. Con esto concepto, podría esbozarse el cuidado de sí desde una fundamentación ética y moral.

Esta relación entre gobierno de sí y el fundamento ético expuesto por los adolescentes como principios o valores morales, representan para ellos, las bases y creencias con los que cuenta cada uno, según sus experiencias vividas. Es por eso que admiten que los principios crecen con la persona en la medida en viven cada acontecimiento. Como lo expresa Deleuze (2015), "gobernarse a sí mismo se despega tanto del diagrama de poder como del código de saber... doble desenganche... la relación con uno mismo, adquiere independencia" (p.98)

La descripción profunda de este individuo ético, es definido por Foucault en tres momentos: el momento de la relación con la verdad, que nos constituye como sujetos de conocimiento; el momento de la relación con el poder, que nos hace establecer una línea propia para la toma de decisiones como sujetos que actúan con otros, y un momento ético, por el que nos instituimos como agentes morales (Foucault, 1984).

En síntesis, develar la cotidianidad de los adolescentes, su pensar, su sentir y su visión del mundo como sujetos, involucra la comprensión de la influencia familiar, social y cultural que han asumido a lo largo de sus vidas, no solo desde la experiencia acontecida, sino lo que involucra en sí misma la acción de construir y deconstruir lo aprendido en cada momento vivido.

Es el reconocimiento de las formas particulares que encuentran para adjudicarle sentido a sus actos, la traducción que hacen de ellos a través del cuerpo en los entornos familiares y sociales y la representación que tienen del cuidado de sí mismos en torno a esas vivencias. Comprender en este punto, el cuidado de sí como eje reflexivo de esta investigación, indica orientar una mirada integral que capte en los relatos de estos jóvenes, sus estrategias de cuidado y la manera en que los otros participan en esa construcción de subjetividades como fundamento de sus realidades cotidianas.

\section{Las normas de los padres}

Las normas o medidas regulatorias para convivencia, según Barrientos (2018), hacen parte de las estrategias parentales que buscan lograr en los hijos, respuestas conductuales apropiadas, por tanto, que sean adoptadas y respetadas por los hijos. Ante ciertas circunstancias, surgen como mecanismos reguladores de los comportamientos que los padres consideran "inadecuados" y de esa forma, se conectan con la acción de cuidar. Son definidas por los adolescentes como:

\section{[...] directrices que indican lo que se debe hacer" (A.d-18 años); "son aquellas pautas que orientan las buenas acciones [...] (A.e-17 años).}

Los jóvenes consideran que no suelen ser las mismas normas que se tenían cuando se era pequeño, al parecer cambian porque las situaciones que ahora viven en la adolescencia, son totalmente diferentes.

[...] las normas cambian, al parecer porque uno también cambia, pero aun así, se deben cumplir [...]

(A.h- 17 años).

Es por ello que son vistos por sus padres como personas con un mayor grado de independencia, criterio para cuidarse y hacer elecciones. Sin embargo, estas precauciones, como también son llamadas por ellos, no siempre son de total agrado, sin embargo, comprenden que son necesarias para mantener el bienestar. 
Esas normas que consideran correctas, que por lo general pueden ser dialogadas, o en cierto grado flexibles, son percibidas de manera positiva por los jóvenes, pues dejan clara la intensión de ser una pauta de cuidado, pero a la vez, de permitirles la libertad para actuar.

[...] cuando uno se porta bien y tiene en cuenta las normas, los papás confían en uno y en lo que uno hace [...] (A.g- 17 años)

[...] cuando me tomo mis tragos y se me va como la mano, llegan los papás y dicen: bueno mijo, que esto no se vuelva una rutina [...] (A.b-18 años).

En esa medida, los padres son considerados por los adolescentes como apoyos que les ayudan a comprender la razón de las normas: más como una guía y no como algo impuesto.

\section{[...] mi mamá es más una líder que una jefe que manda}

y ya; más que cohibirme con las reglas, ella busca que comprenda que es lo mejor para mi"; "Sé que las decisiones que mi mamá toma, no buscan que yo sea un santo o que no tenga vida social, sino que buscan que yo esté bien[...] (A.b- 18 años).

Sin embargo, no siempre el objetivo de las normas busca que sean comprendidas por los jóvenes; en algunas circunstancias suelen ser impuestas, inflexibles o por el contrario, ser tan flexibles al punto de no tener límites, lo que puede beneficiar ciertas conductas de riesgo en los adolescentes; ambas son apreciadas por ellos como poco apropiadas. Las normas que son estimadas como demasiado flexibles pueden presentarse por la falta de claridad y por incumplimiento de las mismas:

[...] los papás no pueden ser tan relajados, como demostrándole al hijo que no les importa lo que pase con él. Hay que tener un punto medio, entre te doy tu libertad pero a la vez te exijo y así te cuido[...] (A.a-17 años).

Bajo esta perspectiva, las normas son mediadas por el poder y el conocimiento que los padres tienen sobre los hijos. El poder juega un papel importante en todas las relaciones, es una estrategia o habilidad de influir o controlar a otros como una posibilidad de moldear, limitar, normalizar y prohibir conductas que son consideradas equivocadas o reprochables social y culturalmente (Fernández, 2018).
El objetivo del poder es dominar bajo patrones de conductas y memorización de discursos que en últimas conducen las prácticas sociales de los sujetos. Permea toda clase de relaciones, circula de un contexto a otro y se modifica a lo largo de los procesos históricos que acontecen en cada sociedad o sistema relacional.

De esta manera sería posible comprender que las familias modulan el poder de acuerdo a su propia historia y acontecimientos, que van mutando en cada etapa del ciclo vital según los reajustes que consideran necesarios para mantener el orden familiar. El otro caso sería cuando ocurre lo contrario, normas inflexibles y rígidas son apreciadas por los adolescentes como aquellas que son exigentes e impuestas por los padres, que no admiten discusión alguna.

[...] es que si a uno le imponen las cosas, uno no se cuida, uno se rige bajo algo que le impusieron, más no por algo de lo cual se piensa y se analiza; eso no es cuidar [...] (A.a-17 años).

Son normas escudadas en la intensión de cuidar a los hijos de "las cosas malas" que les pueden ocurrir en la calle, y se pasa por alto, que someterlos de esa forma suele conducirlos posiblemente a la toma de decisiones equivocadas.

[...] A los jóvenes que cuidan mucho, cuando los papás los dejan salir, quieren hacer lo que no han podido hacer en mucho tiempo, entonces mientras más los cohíban, más van a querer hacer las cosas que no deben [...] (A.g- 18 años)

Consideran además que:

[...] un hijo que no lo dejen salir, que sean totalmente intransigentes con él, se va a cansar de eso y se va a volver rebelde y esa rebeldía lo puede llevar a los vicios y a las malas conductas [...] (A.a-17 años).

Ante esta dinámica inflexible, el adolescente emprende el camino hacia la independencia y busca alejarse del dominio parental, dado que está presto a "salir" del nido familiar para encaminar la búsqueda en el mundo exterior. La familia, simultáneamente, se enfrenta a un periodo de separación de los hijos, lo que para algunos padres, suele ser un proceso difícil de comprender y aceptar. Serán los vínculos existentes entre ambos, los que determinen el avance o el estancamiento de la interacción familiar. 
En este sentido, Sánchez, Londoño, Lozano y Chavarria (2015) describen en su trabajo investigativo que la estructura familiar está definida por la interacción de los sujetos, la cual se reajusta ante las nuevas situaciones acontecidas en cada etapa de su ciclo vital.

Por tal razón, en las situaciones de tensión y divergencia, los padres buscan mantener el control y el equilibrio en las relaciones con sus hijos; por tanto, los acuerdos, arreglos y transacciones se convierten en la piedra angular para conservar la seguridad y estabilidad de las relaciones parentales. Pero contrario a los patrones de control, Blanco y Licona (2016) confieren que un patrón parental que promueva y acompañe a sus hijos en la toma de decisiones, colabore en la construcción de esa toma de decisiones y las acepte, es promulgar por interacciones exitosas que llegan a crear vínculos parentales fuertes y de confianza.

Se afirma también que en esta etapa es importante que los padres logren consensuar actividades, tiempos, roles y responsabilidades con sus hijos, además de fortalecer los canales de comunicación entre la misma pareja, dado que esto es percibido por los hijos como un factor protector y de bienestar para ellos (Ortega, Plancarte, Garrido, Reyes y Torres, 2018). No obstante se ha tenido el pensamiento social que los padres son las personas capaces y capacitadas para estructurar el entorno familiar de los hijos, responsables de crear relaciones de confianza y vínculos afectivos adecuados que den respuesta a las situaciones desequilibradas que surgen de la interacción familiar.

De esta manera el ejercicio parental se centra en la conducta del adolescente como mecanismo para justificar acciones de control, dado que el adolescente ratifica su conducta en la posibilidad de vivir la experiencia como proceso natural de aprendizaje, la cual, resulta ser más significativa que la misma sujeción a la norma parental.

En una dimensión ideal como lo expresan los jóvenes, las relaciones con los padres deberían ser aquellas que consideran la libertad como una práctica reflexiva, es decir como la oportunidad para actuar libremente sin ser juzgado.
Es así como los sujetos deben ocuparse de sí mismos para que sus pensamientos y acciones, estén en consonancia permanente con sus vivencias. Es un camino que el individuo recorre no en solitario de los otros, sino en solidaridad con su propia existencia. Por lo tanto, el hecho de cuidar de los otros -como es en el caso de los padres- no es por sí sola una posibilidad de establecer pautas apropiadas de cuidado en los jóvenes, es justamente el hecho de comprender que los cambios sobrevienen con o sin su apoyo, que estarán indiscutiblemente expuestos a los riesgos, pero que una relación parental de confianza, dispuesta al diálogo permanente, será para el adolescente una mecanismo de soporte para sobrellevar de una manera más adecuada sus aprendizajes (Rodríguez, Echeverría, Alamilla y Trujillo, 2018; Romero y Acosta, 2018).

Esta sería tal vez la denominación adolescente de una relación parental ideal: aquella en la que puedan expresar sus pensamientos, actuar sin temor a la ocurrencia del error o al reproche de los padres. Una relación donde precisamente no sea el señalamiento lo que prime, sino la comprensión; la benevolencia por un sujeto que puede aprender de sus propias equivocaciones y que busca en medio de sus recursos, transformarse, rehacerse y trasegar nuevos caminos que lo conduzcan a cuidar de sí mismo.

\section{Conclusiones}

Los adolescentes aprenden a través de sus propias experiencias a cuidar de sí mismos. Las enseñanzas recibidas por los padres hacen parte de su sistema de valores que se pone a prueba en cada acontecimiento de la vida en la que deban tomar decisiones para su propio cuidado. En la medida en que la conducta esté alineada a la norma y al sistema de valores, las relaciones con los padres son tranquilas y por ende de confianza.

No obstante, los jóvenes se ven enfrentados a vivir situaciones que los conducen a comportarse de manera diferente a lo establecido; es ahí donde este desvío de lo pautado y regulado por los padres se concibe para la mayoría de ellos como error, es decir, una falta al propio cuidado. Esta situación de riesgo, hará que la dinámica normativa sea puesta en marcha como una posibilidad para aprender del error. 
En tanto que para los adolescentes la confianza manifiesta por los padres es el elemento fundante de las relaciones parentales, la negociación de las normas y la comunicación permanente, se suman a estas prácticas filiales; empero, no dejan de reconocer que sus contextos cercanos y las circunstancias sociales actuales, suelen ser favorecedoras de conductas de riesgo y determinan un "forcejeo" constante por mantenerse en el umbral de la acción correcta, el cumplimiento de la norma y el respeto por sí mismos y los otros.

Las limitaciones del presente estudio están dadas por las características de la muestra, pues esta podría no ser representativa de los entornos escolares de áreas diferentes a la urbana, donde es posible que la población posea otras características socioculturales distintas, y a su vez que ello pueda otorgar una mayor variabilidad a los resultados. Asimismo, el estudio no contempló la inclusión de los padres, lo que podría ser una consideración para futuras investigaciones.

\section{Agradecimientos}

Al señor Absalón Arcila Arias Rector del colegio y a cada uno de los jóvenes por sus voces y momentos compartidos.

\section{Conflicto de Intereses}

Declaro no tener ningún tipo de conflicto de intereses, ni ninguna relación política, personal o financiera que haya influido en los resultados de esta investigación.

\section{Referencias}

Alzate, M. C. E. (2014). Procedimiento de familia y del menor. Bogotá: Leyer.

Barrientos, A. M. R. (2018, julho). Prácticas adolescentes: el cuidado de sí en las relaciones parentales. Atas CIAIQ 2018. Fortaleza, CE, Brasil, 7. Recuperado de https:// proceedings.ciaiq.org/index.php/ciaiq2018/article/ view/1723/1676

Berger, K.S. (2016). Psicología del desarrollo infancia y adolescência ( $9^{\mathrm{a}}$ ed.). Madrid: Editorial Médica Panamericana.
Blanco, A. I., \& Licona, J. F. M. (2016). Concepciones sobre la crianza: El pensamiento de madres y padres de familia. Liberabit, 22(1), 31-41. Recuperado de http://www. scielo.org.pe/scielo.php?script=sci_arttext\&pid=S1729$48272016000100003 \&$ lng $=$ es\&tlng=es

Castillo, S., Sibaja, D., Carpintero, L., \& Romero-Acosta, K. (2015). Estudio de los estilos de crianza en los niños, niñas y adolescentes en Colombia: un estado del arte. Búsqueda, (15), 64-71. Recuperado de http://revistasolds.cecar.edu. co/busqueda/article/viewFile/97/90

Cogollo, Z., \& Gómez-Bustamante, E. M. (2013). Health risk behavior pattern among students from Cartagena, Colombia: prevalence and associated variables. Revista da Escola de Enfermagem da USP, 47(4). 830-835. doi: 10.1590/S0080-623420130000400009

Deleuze, G. (2015). La subjetivación: curso sobre Foucault (Tomo III). Buenos Aires: Editorial Cactus.

Díaz-Alzate, M. V., \& Mejía-Zapata, S. I. (2018). La mirada de los adolescentes al modelo de habilidades para la vida. Revista Latinoamericana de Ciencias Sociales, Niñez y Juventud, 16(2), 709-718. doi: 10.11600/1692715x.16205

Foucault, M. (1978). La verdad y las formas jurídicas. Barcelona: Editorial Gedisa.

Foucault, M. (1983), El sujeto y el poder. Barcelona: Carpe Diem Ediciones.

Foucault, M. (1990), Tecnologías del yo y otros textos afines. Barcelona: Ediciones Paidós Ibérica S.A.

Foucault, M. (1994). Estética, ética y hermenéutica. Obras esenciales volumen III. Barcelona: Paidós.

Foucault, M. (2001). La hermenéutica del sujeto. Buenos Aires: Fondo de cultura económica.

Foucault, M. (2011). Historia de la sexualidad 2: el uso de los placeres (2a ed.). Ciudad de México: Siglo Veintiuno Editores.

Fernández, R. G. (2018). Hacia una construcción del sujeto en Michel Foucault. Revista electrónica de estudiantes de la Escuela de Psicología de la U.C.R., 13(1). 9-26. Recuperado de https://dialnet.unirioja.es/servlet/ articulo?codigo $=6345900$

Güemes-Hidalgo, M., González-Fierro, M. J. C., \& Vicario, M. I. H. (2017). Pubertad y adolescencia. Revista Adolescere, 5(1), 7-22. Recuperado de https://www.adolescenciasema.org/ tema-de-revision-pubertad-y-adolescencia-m-guemeshidalgo-et-al-adolescere-2017-v-1-07-22/

Hallal, R. (1998). Cuidado de uno mismo: nostalgia o novedad?. Adolescência latinoamericana, 1(1). 
Henao, A. M. G. (2012). Recuperación crítica de los conceptos de familia, dinámica familiar y sus características. Revista virtual Universidad Católica del Norte, (35). 326345. Recuperado de http://www.redalyc.org/articulo. oa?id=194224362017

Hernández, E. (2018). Estado de la investigación sobre jóvenes en Bogotá, Colombia y América Latina. Infancias Imágenes, 17(2), 185-196. Recuperado de https://dialnet.unirioja.es/ servlet/articulo?codigo $=6633297$

Medina, P. Q., Álvarez, A. A., \& Hernández, V. M. H. (2017). Comunicación entre padres e hijos adolescentes. Jóvenes en la ciencia, 3(2), 432-437. Recuperado de https://www. researchgate.net/publication/326380550_Comunicacion_ entre_padres_e_hijos_adolescentes

Ortega, P., Plancarte, P., Garrido, A., Reyes, A. \& Torres, L. (2018). Una visión de alumnos universitarios: relación de padres como pareja. Acta colombiana de Psicología, 21 (2), 88-98. doi: 10.14718/ACP.2018.21.2.5

Plasín. M. J. E., \& Goicochea, M. A. D. (2012). Adolescentes de hoy: aspiraciones y modelos. Madrid: Liga Española de la Educación. Recuperado de http://ligaeducacion.org/wpcontent/uploads/2018/03/adolescentes-de-hoy.pdf

Restrepo, E. (2016). Etnografía: Alcances, técnicas y éticas. Bogotá: Envión editores.

Rodríguez, S. G. A., Echeverría, R. E., Alamilla, N. M. E., \& Trujillo, C. D. C. (2018). Prevención de factores de riesgo en adolescentes: intervención para padres y madres. Psicología Escolar e Educacional, SP. 22(2), 259-269. doi: $10.1590 / 2175-35392018014279$

Romero, S. L G., \& Acosta, V. E. Z. (2018). Comunicación activa entre padres-madres e hijos-hijas (Trabalho de conclusão de curso). Facultad de Psicología, Universidad Católica de Colombia, Bogotá, Colombia. Recuperado de https:// repository.ucatolica.edu.co/handle/10983/22262

Rojas Betancur, M., Méndez Villamizar, R., \& Álvarez Nieto, C. (2016). El papel de la familia en la normalización del embarazo a temprana edad. Revista Encuentros, 14(1), pp.139-150. doi: $\underline{10.15665 / \text { re.v14i1.674 }}$

Sánchez, G. D., Londoño, M. A., Lozano, N. Y., \& Chavarria, E. V. (2015). Sobre la dinámica familiar. Revisión documental. Cultura Educación y Sociedad, 6(2), 117-138. Recuperado de https://revistascientificas.cuc.edu.co/ culturaeducacionysociedad/article/view/1049

Villalba, T.M. (2015). El bienestar de los adolescentes en las modernas estructuras Familiares (Tese de doutorado). Universidad Complutense de Madrid, Madrid, España. Recuperado de https://eprints.ucm.es/43329/1/T38928. pdf 